\title{
Chaetotaxy of the monogeneans Macrogyrodactylus clarii and M. congolensis from the gills and skin of the catfish Clarias gariepinus in Egypt, with a note on argentophilic elements in the nervous system
}

\author{
Mohammed M. El-Naggar ${ }^{1}$, Safaa Z. Arafa ${ }^{1}$, Sameer A. El-Abbassy $^{1}$ and Graham C. Kearn $^{2}$ \\ ${ }^{1}$ Zoology Department, Faculty of Sciences, Mansoura University, Mansoura, Egypt; \\ ${ }^{2}$ School of Biological Sciences, University of East Anglia, Norwich NR4 7TJ, UK
}

Key words: Monogenea, Macrogyrodactylus clarii, Macrogyrodactylus congolensis, surface sensilla, nervous system, chaetotaxy, Clarias gariepinus

\begin{abstract}
A comparison has been made between the chaetotaxy of the gyrodactylid monogeneans Macrogyrodactylus clarii Gussev, 1961 and M. congolensis (Prudhoe, 1957) Yamaguti, 1963 from the gills and skin, respectively, of the catfish Clarias gariepinus (Burchell) from the river Nile in Egypt. Bilaterally arranged argentophilic structures on the surface of these parasites are presumed to be sensilla and are more abundant in M. clarii than in M. congolensis especially on the ventral surface (124 vs. 66). In both species these sensilla are concentrated on the head lobes and in the pharyngeal region, but there are features of the sensilla patterns that can be used to distinguish the two species. Comparison is made with sensilla patterns of other gyrodactylids. A system of cells and dendritic processes, most probably part of the nervous system, also has an affinity for silver in the two species. There are no previous records of extensive argentophilic elements in the nervous systems of monogeneans.
\end{abstract}

The silver nitrate staining technique of Lynch (1933) is an effective way of visualising cell junctions on the surface of monogeneans. These animals are covered by a syncytial tegument, which is not argentophilic. However, wherever cellular structures breach the tegument, the intercellular material between the tegumental limiting membrane and the cell membrane of the intruding structure appear to have strong affinities for silver. Intruding cellular structures include ciliated or unciliated nerve endings, which are presumed to be sensory (sensilla), gland duct terminations and openings of the excretory system, reproductive system and gut. Most previous studies have concentrated on the distribution of sensilla in monogenean larvae (oncomiracidia) (see, for example, Combes and Lambert 1972, Lambert 1977a, b, 1978a, b, Tinsley 1978). Studies of the distribution patterns of surface sensilla in adult monogeneans are few (Lambert 1979, El-Naggar et al. 1993, Khidr and El-Naggar 1996, Shinn et al. 1997, 1998a, b). Chaetotaxy maps have been used by some authors (Maeder 1973, Shinn et al. 1997, 1998a, b) as a diagnostic tool for the discrimination of different species of monogeneans. The finding in Egypt of Macrogyrodactylus clarii Gussev, 1961 on the gills and M. congolensis (Prudhoe, 1957) Yamaguti, 1963 on the skin of the catfish, Clarias gariepinus (Burchell), provided an opportunity to compare the chaetotaxy of two related gyrodactylids occupying different microhabitats on the same host.

\section{MATERIALS AND METHODS}

Specimens of the catfish Clarias gariepinus were caught from the Demietta branch of the river Nile near Mansoura, Egypt. Fishes were kept alive until required in a tank containing aerated river water. The head of each fish was cut using a knife blade and the whole body of the fish including the head was placed in dissecting dish containing river water. The gills were removed and searched for Macrogyrodactylus clarii and the fins and body surface were also examined for $M$. congolensis.

The silver nitrate staining technique of Lynch (1933) was applied to about 20 living, unflattened specimens of each species. Living parasites were washed several times in river water and expelled from a pipette into a shallow dish containing hot $\left(70-80^{\circ} \mathrm{C}\right) 0.5-1 \%$ silver nitrate solution. The parasites were then placed beneath a tungsten lamp for about $15 \mathrm{~min}$. When argentophilic features of the parasites were sufficiently well defined, the parasites were removed and washed in several changes of distilled water. They were dehydrated in an ascending series of ethanol, cleared in xylol, mounted in Canada balsam and examined using the light microscope.

\section{RESULTS}

After exposure of silver-nitrate-treated parasites to light, small dark brown rings, assumed to be sensilla, appear on their surfaces. In each species, the distribution of these sensilla is bilaterally symmetrical. The most common distribution patterns in M. clarii and in M. con- 


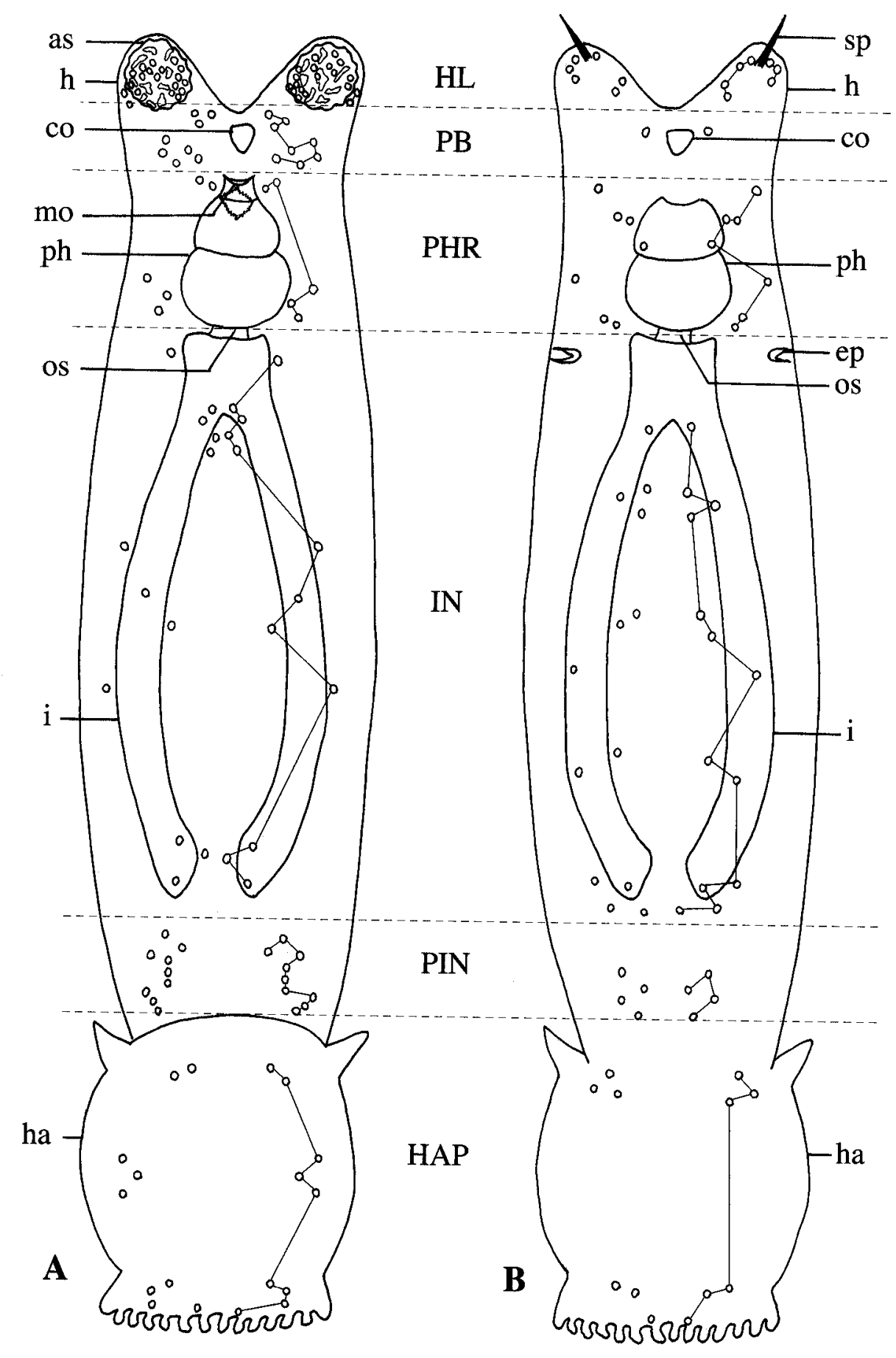

Fig. 1. Diagram showing the distribution pattern of surface sensilla of Macrogyrodactylus clarii. A - ventral view; B - dorsal view. Note that the dashed lines divide the body into six regions, namely head lobe region (HL), pre-buccal region (PB), pharyngeal region (PHR), intestinal region (IN), post-intestinal region (PIN) and haptoral region (HAP). as - adhesive area; co cerebral organ; ep - excretory pore; ha - haptor; $\mathrm{h}$ - head lobe; $\mathrm{i}$ - intestine; mo - mouth opening; os - oesophagus; ph pharynx; sp - spike. Scale bar $=250 \mu \mathrm{m}$.

golensis are shown in Figs. 1 and 2, and the total numbers of sensilla in different regions of the parasites are compared in Table 1. In a few individuals the number and distribution of the sensilla deviated slightly from this pattern. It should be noted that the lines in
Figs. 1 and 2 linking the sensilla in groups and the divisions of the body of the parasites into six regions (HL, head lobe; PB, pre-buccal; PHR, pharyngeal; IN, intestinal; PIN, post-intestinal; HAP, haptoral) are for the convenience of description and do not necessarily 


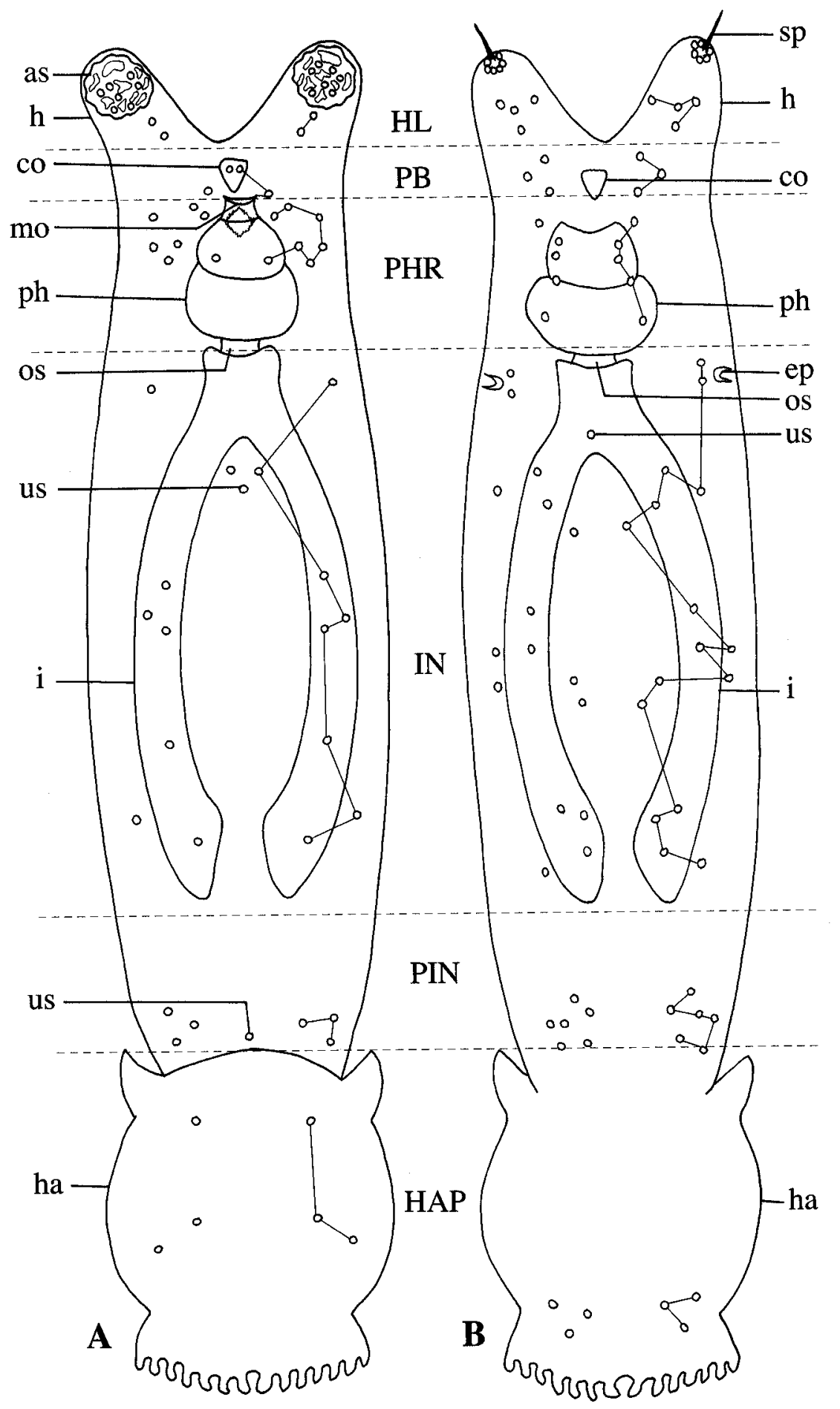

Fig. 2. Diagram showing the distribution pattern of surface sensilla of Macrogyrodactylus congolensis. A - ventral view; B dorsal view. us- unpaired sensillum. Other abbreviations as in Fig. 1 . Scale bar $=250 \mu \mathrm{m}$.

imply that the sensilla of each group are functionally related.

The total number of sensilla on the ventral surface of M. clarii (Fig. 1A, Table 1) is 124. They are distributed on each side as follows: head lobe region 19 sensilla (2 outside and 17 inside the anterior adhesive area); prebuccal region 8 sensilla; pharyngeal region 5 sensilla; intestinal region 12 sensilla; post-intestinal region 9 sensilla; haptoral region 9 sensilla.

The total number of sensilla on the dorsal surface of M. clarii (Fig. 1B, Table 1) is 76. They are distributed on each side as follows: head lobe region 7 sensilla, together with a relatively large and conspicuous compound sensory structure or "spike" organ; pre- 
buccal region one sensillum; pharyngeal region 7 sensilla; intestinal region 13 sensilla; post-intestinal region 4 sensilla; haptoral region 6 sensilla.

The total number of sensilla on the ventral surface of M. congolensis (Fig. 2A, Table 1) is 66. This includes 2 unpaired sensilla located in the mid-line, one posterior to the intestinal bifurcation and one just anterior to the haptor. The rest of the ventral sensilla are paired and are distributed on each side of the body as follows: head lobe region 9 sensilla ( 2 outside and 7 inside the anterior adhesive area); pre-buccal region 2 sensilla; pharyngeal region 7 sensilla; intestinal region 8 sensilla; postintestinal region 3 sensilla; haptoral region 3 sensilla.

The dorsal surface of $M$. congolensis accommodates 87 sensilla (Fig. 2B, Table 1). This includes a single unpaired sensillum located in the mid-line near the intestinal bifurcation. All the other dorsal sensilla are paired and are distributed on each side of the body as follows: head lobe region 10 sensilla, plus a spike-organ similar to that of M. clarii (6 of the ring sensilla are closely associated with the spike); pre-buccal region 3 sensilla; pharyngeal region 5 sensilla; intestinal region 16 sensilla; post-intestinal region 6 sensilla; haptoral region 3 sensilla.

Some other surface features that are generally larger than the presumed sensilla are also revealed by Lynch's technique. The mouth opening in $M$. clarii and $M$. congolensis appears as a slit on the ventral side of the head region and, within the buccal cavity of both parasites, there is a zigzag, silver-stained feature forming a circle (Figs. 1A, 2A, 4). The excretory pores of $M$. clarii and $M$. congolensis are visible as crescentic stained features, one on each dorso-lateral side of the body at the level of the oesophagus (Figs. 1B, 2B). Each anterior head lobe of $M$. clarii and $M$. congolensis bears an adhesive sac and the edge of the ventrally directed opening of this sac is argentophilic (Figs. 1A, 2A). Within the sac there are, in addition to the regular circular sensilla mentioned above, numerous slightly larger, silver-stained features with more irregular, less strictly circular outlines.

Argentophilic elements inside the body were also revealed by Lynch's technique. In particular, an extensive, bilaterally symmetrical, dendritic pattern of cells and cell processes, which seems most likely to be part of the nervous system, is apparent in $M$. clarii (Fig. 3A) and in M. congolensis (Figs. 3B, 4-6). In M. clarii there are five cell bodies located dorsally on each side of the animal and these cell bodies are linked by a longitudinally running nerve process and by side branches (Fig. 3A, d1-d5). There are 3 cells on each side ventrally (Fig. 3A, v1-v3), which are similarly linked by a longitudinal process, and there are at least 3 cross-links between the dorsal and ventral systems. Dendritic processes extend the dorsal and the ventral system in a median direction and in some specimens links between the longitudinal systems on opposite sides of the body were seen.

A similar pattern of cells and connections occurs in $M$. congolensis (Figs. 3B, 4-6). The presumed nerve cells contain spherical granules (Fig. 6). In addition, $M$. congolensis possesses two adjacent argentophilic cells on each side close to the haptor, with which each lateral pair of cells communicates by a common argentophilic process (Fig. 3B). Apart from this process, no other argentophilic structures were located in the haptor or in the region anterior to the pharynx where the "brain" is likely to be located. The so-called "cerebral organ" (Fig. 3; Prudhoe 1957, El-Naggar and Serag 1987) has no affinity for silver and has no connections with the argentophilic dendritic system.

\section{DISCUSSION}

The silver impregnation technique of Lynch (1933) has been used to reveal the positions of argentophilic rings, assumed to indicate the presence of sensilla, on the surfaces of two gyrodactylid (monogenean) parasites, Macrogyrodactylus clarii from the gills and $M$. congolensis from the skin of the catfish Clarias gariepinus. The technique also revealed the locations of the excretory pores, the mouth and the openings of the anterior adhesive sacs on the head lobes. Inside the adhesive sacs silver-stained features that were slightly larger and more irregular than the strictly circular sensilla are probably the openings of gland ducts. There is also a zigzag, silver-stained, roughly circular pattern visible inside the mouth. The nature of this feature will be considered below.

Chaetotaxy patterns for Macrogyrodactylus spp. have not previously been published, although species of the related genera Gyrodactylus and Ooegyrodactylus have been studied (Shinn et al. 1997, 1998a, b). As in other gyrodactylids, the sensilla patterns of Macrogyrodactylus are bilaterally symmetrical, but the sensilla are not evenly distributed, being concentrated in conspicuous groups in some areas and scattered or absent in others. It has been the practice to link neighbouring sensilla in groups since this aids comparison with other parasites.

The sensilla patterns of the viviparous Gyrodactylus spp. and the oviparous Ooegyrodactylus farlowellae are similar, indicating a close relationship between them according to Shinn et al. (1998a). The sensilla patterns of Macrogyrodactylus spp., which are also viviparous, differ in some important respects from those of these other gyrodactylids. If it is accepted that sensilla patterns are reliable indicators of relationships, then the implication is that Gyrodactylus and Ooegyrodactylus are more closely related to each other than either of these genera is to Macrogyrodactylus. A striking feature of the sensilla patterns of Gyrodactylus and Ooegyrodactylus is the presence in the mid-ventral region of 
El-Naggar et al.: Chaetotaxy of Macrogyrodactylus spp.
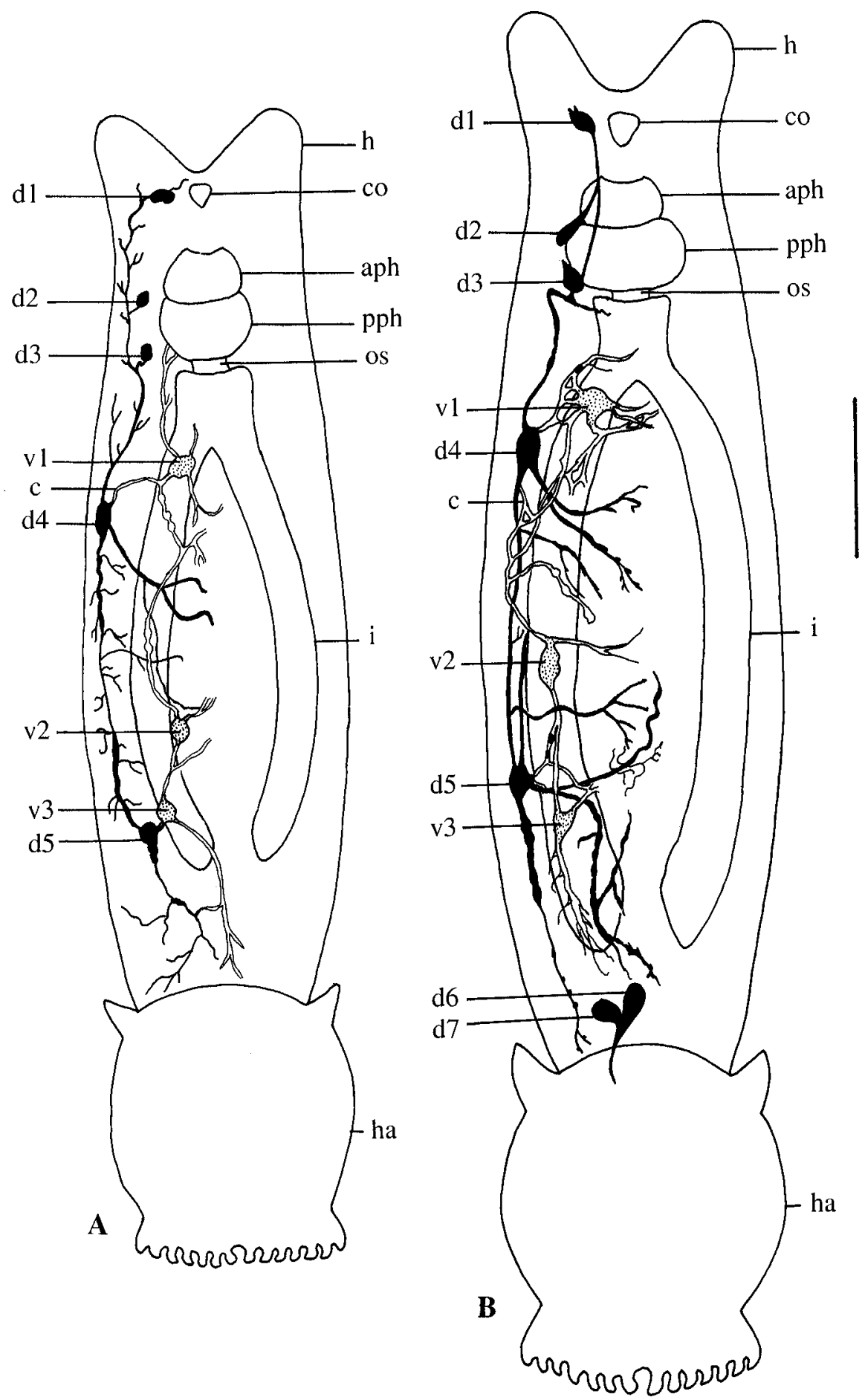

Fig. 3. Diagram showing internal argentophilic system (part of the nervous system?). A - Macrogyrodactylus clarii; B - M. congolensis. System shown on one side of the body only; dorsally situated cells (d1-d7) and connectives black; ventrally situated cells (v1-v3) stippled and connectives unshaded. aph - anterior region of the pharynx; c - cross-link; co - cerebral organ; $\mathrm{h}$ head lobe; ha - haptor; i - intestine; os - oesophagus; $p$ ph - posterior region of the pharynx. Scale bar $=250 \mu \mathrm{m}$.

the body of two parallel longitudinal rows, each of 3 large, equally spaced sensilla. Shinn et al. (1998a) observed that a similar arrangement of sensilla $(3 \times 2)$ occurs in oncomiracidia of polyopisthocotyleans and, even though these sensilla are dorsally located, they interpreted this similarity as evidence that gyrodactylids are closer to polyopisthocotyleans than previously thought. This distinctive pattern of $3 \times 2$ ventral sensilla is not present in Macrogyrodactylus, casting some doubt on the interpretation of Shinn et al. 


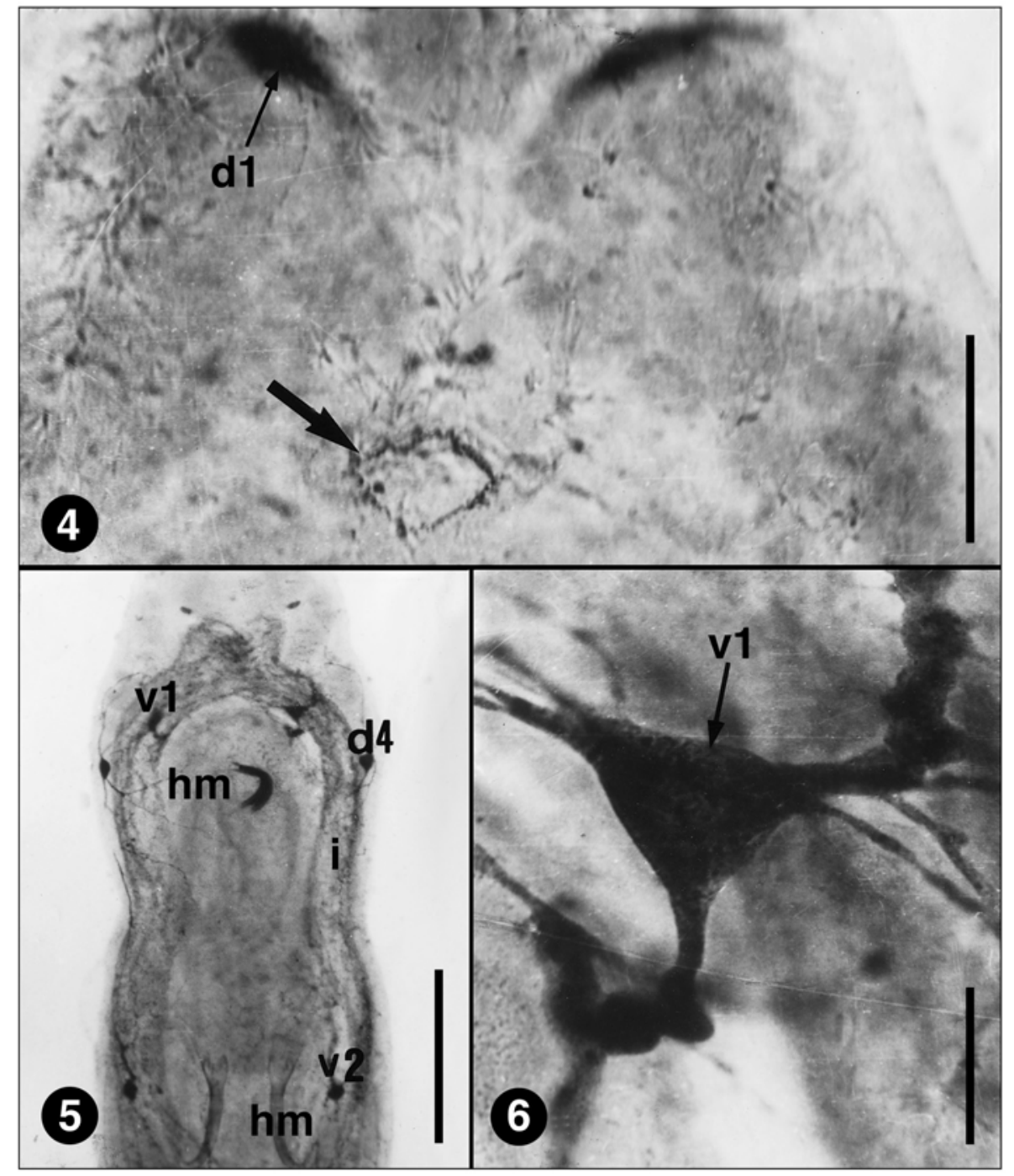

Figs. 4-6. Photomicrographs of whole-mount specimens of Macrogyrodactylus congolensis stained with silver nitrate. Fig. 4. The head region showing the roughly circular (zigzag) feature within the buccal cavity (arrow) and an argentophilic (nerve?) cell (d1 in Fig. 3B). Fig. 5. The middle region of the body showing argentophilic (nerve?) cells d4, v1 and v2. Hamuli (hm) are visible in two embryos; i - intestine. Fig. 6. Argentophilic (nerve?) cell (v1 in Fig. 3B). Scale bars: Fig. $4=25 \mu \mathrm{m} ;$ Fig. $5=250$ $\mu \mathrm{m}$; Fig. $6=10 \mu \mathrm{m}$.

Clusters of sensilla around or close to the excretory pore are also features of Gyrodactylus and Ooegyrodactylus (see Shinn et al. 1998a). In M. clarii no sensilla were found near the excretory pore and in $M$. congolensis only two sensilla were in the vicinity of the pore. Macrogyrodactylus has more sensilla than Gyrodactylus and Ooegyrodactylus but is significantly larger.

There are many differences between the sensilla patterns of M. clarii and M. congolensis. First, M. clarii has more sensilla (200) than M. congolensis (153). This difference can be mainly attributed to greater numbers of ventral sensilla in M. clarii, especially on the head lobes and in the pre-buccal, post-intestinal and haptoral regions (Table 1). It is unlikely that this feature is related to body size, since $M$. clarii and $M$. congolensis are similar in size. One possibility is that the enhanced number of ventral sensilla in $M$. clarii may be a reflection of its gill-dwelling habit. Since $M$. clarii seems likely to move from the gills to the skin prior to changing hosts, it would be of interest to determine whether this change of microhabitat is associated with any corresponding change in number or pattern of sensilla.

Differences between M. clarii and M. congolensis in number and distribution of surface sensilla could be used, in addition to anatomical and morphological differences (El-Naggar and Serag 1987, El-Naggar et. al. 1999), to discriminate between them, particularly, 
Table 1. Total number of sensilla on different regions of the dorsal and ventral surfaces of Macrogyrodactylus clarii and M. congolensis. HL - head lobe region; PB - pre-buccal region; PHR - pharyngeal region; IN - intestinal region; PIN - postintestinal region; HAP - haptoral region.

\begin{tabular}{|l|c|c|c|c|c|c|c|c|}
\hline Species & Body surface & HL & PB & PHR & IN & PIN & HAP & Total \\
\hline M. clarii & ventral & 38 & 16 & 10 & 24 & 18 & 18 & 124 \\
M. congolensis & ventral & 18 & 4 & 14 & 17 & 7 & 6 & 66 \\
M. clarii & dorsal & 14 & 2 & 14 & 26 & 8 & 12 & 76 \\
M. congolensis & dorsal & 20 & 6 & 10 & 33 & 12 & 6 & 87 \\
\hline
\end{tabular}

when these parasites are infesting the same host fish. Maeder (1973) and Combes and Lambert (1975) used chaetotaxy to differentiate between polystomatid larvae and Shinn et. al. (1997) used chaetotaxy maps to differentiate between species of the genus Gyrodactylus. Important features that are likely to be useful in discriminating between Macrogyrodactylus clarii and $M$. congolensis are differences in the number and pattern of haptoral sensilla on both the dorsal and ventral surfaces. However for Gyrodactylus spp., Shinn et al. (1997) did not consider the haptoral pattern of sensilla sufficiently reliable for use in discriminating between species.

The presence of presumed sensilla on the ventral surface of the haptor in Macrogyrodactylus (and in related gyrodactylids; see Shinn et al. 1997, 1998a) is of special interest. In the capsalid skin parasite Entobdella soleae cilia-based sensilla have not been located on this surface, although papillae that may be sensory are present (Kearn 1993). Sensilla on the ventral surface of the haptor may be permanently in contact with the host's skin, unlike sensilla elsewhere on the body, and their continued stimulation may be important to maintain attachment. On the other hand, they may only function during leech-like locomotion, perhaps registering renewed contact with the host at the end of a locomotory "step" and initiating the deployment of the hooks. However, it has not yet been established that these argentophilic haptoral rings are cilia-based sensilla. It is possible that some may be gland duct openings or, as Shinn et al. (1997) pointed out, the silver-stained apertures through which the points of the marginal hooklets and hamuli protrude. Examination of these structures with the scanning and transmission electron microscopes is desirable.

The presence in $M$. congolensis of unpaired sensilla in a median position both on the ventral surface (near the intestinal bifurcation and just anterior to the haptor) and on the dorsal surface (near the intestinal bifurcation) is a useful feature for distinguishing this parasite from M. clarii. Unpaired sensilla have been recorded in other gyrodactylids, for example in the haptors of Gyrodactylus derjavini (see Shinn et al. 1997) and Ooegyrodactylus farlowellae (see Shinn et al. 1998a).

During the course of this study it was surprising to find that internal elements of the nervous system had a strong affinity for silver in both species of Macro- gyrodactylus. In spite of the intensive use of Lynch's method of silver impregnation for revealing sites of sensilla on the bodies of larval and adult freshwater monogeneans, there have been no reports, as far as we are aware, of staining of nerve cells and dendrites within the body. However, the Golgi technique, which also involves silver impregnation, has been widely and successfully used for staining nerves in platyhelminths (Bullock and Horridge 1965).

According to Switzer (2000), histological protocols using silver have been used to visualise a wide range of structures or features in biology, including reticulin, melanin, fungi, chromosome bands, nucleolar organising regions and nerves. Peters $(1955 \mathrm{a}, \mathrm{b})$ and Baker (1958) regarded silvering as a two-stage process. In the first of these processes (impregnation), a silver salt is reduced to microscopic "nuclei" of metallic silver, not yet large enough to be seen. In the second process (development), excess unreduced silver is reduced in turn and deposited around the nuclei until they become visible. However, Switzer (2000) provided a brief historical outline of the development of neurohistological silver methods and made the point that, in spite of considerable efforts, the mechanism of staining is still poorly understood. He suggested that, in nerves, amino acids, especially those with single sulphide groups, formed complexes with silver and that the greater affinity of degenerating nerves for silver reflected the disintegration of nerve proteins and the release of more target amino acids.

Whatever the reducer is in Macrogyrodactylus, it appears to be restricted to only part of the nervous system, since there are undoubtedly nervous elements elsewhere in the body (for example in the "brain", in the haptor and in the highly sensitive head lobes) that are not argentophilic. It is also worth noting that silver associated with the surface sensilla is localised in the intercellular matrix between the sensilla and the tegument and not in the nerve process supplying the sensillum. A more complete picture of the nervous system of Macrogyrodactylus will require the application of more modern techniques, such as immunocytochemistry and confocal microscopy (see Johnston et al. 1990). It may also throw light on the reasons for the regional differences in argentophilia in the nervous system of Macrogyrodactylus. 
There are other possible reducing agents, for example phenolic compounds (see Baker 1958), and there is no reason to believe that the agent responsible for silver deposition at the sensillum/tegument junction is the same as the agent that is operative in the nervous elements of Macrogyrodactylus. It is suggested that the line of deposited silver corresponding with the mouth opening of Macrogyrodactylus and the zigzag line of deposited silver deeper inside the mouth, may represent boundaries where one syncytium or lining cell joins another. In other words, there may be a buccal lining that is distinct and separate externally from the body tegument and internally from a pre-pharyngeal lining. This will require confirmation with the transmission electron microscope.

\section{REFERENCES}

BAKER J.R. 1958: Principles of Biological Microtechnique A Study of Fixation and Dyeing. Methuen \& Co., London, $357 \mathrm{pp}$.

BULLOCK T.H., HORRIDGE G.A. 1965: Structure and Function in the Nervous Systems of Invertebrates. Vol. 1. W.H. Freeman \& Co., San Francisco and London, 798 pp.

COMBES C., LAMBERT A. 1972: Evolution de l'appareil sensoriel au cours de la vie chez les Monogènes Polyopisthocotylea. C. R. Acad. Sci., Paris 274, Serie D: 402404.

COMBES C., LAMBERT A. 1975: La chétotaxie des larves nageantes de Polystomes européens (Monogenea). Ann. Parasitol. Hum. Comp. 50: 25-37.

EL-NAGGAR M.M., KEARN G.C., HAGRAS A.E., ARAFA S.Z. 1999: On some anatomical features of Macrogyrodactylus congolensis, a viviparous monogenean ectoparasite of the catfish Clarias gariepinus from Nile water. Journal of the Egyptian German Society of Zoology 29 (D), Invertebrate Zoology and Parasitology: 1-24.

EL-NAGGAR M.M., REDA E.S., ARAFA S.Z. 1993: Observations on the anatomy and surface sensilla of Pseudodactylogyrus anguillae (Yin and Sproston, 1948) Gussev, 1965, a monogenean gill parasite of Anguilla anguilla in Egypt. Journal of the Egyptian German Society of Zoology 11 (D), Invertebrate Zoology and Parasitology: 147-175.

EL-NAGGAR M.M., SERAG H.M. 1987: Redescription of Macrogyrodactylus clarii Gussev 1961, a monogenean gill parasite of Clarias lazera in Egypt. Arab Gulf J. Sci. Res., Agric. Biol. Sci. 5: 257-271.

JOHNSTON C.F., SHAW C., HALTON D.W., FAIRWEATHER I. 1990: Confocal scanning laser microscopy and helminth neuroanatomy. Parasitol. Today 6: 305-308.

KEARN G.C. 1993: Environmental stimuli, sense organs and behaviour in juvenile/adult monogeneans. Bull. Fr. Pêche Piscic. 328: 105-114.

KHIDR A.A., EL-NAGGAR M.M. 1996: Observations on the anatomy and surface sensilla of the monogenean gill parasite Cichlidogyrus arthracanthus Paperna, 1960 from Tilapia spp. in Egypt. Journal of the Egyptian German Society of Zoology 19 (D), Invertebrate Zoology and Parasitology: 21-41.

LAMBERT A. 1977a: Développement larvaire et post-larvaire d'Ergenstrema mugilis Paperna, 1964 (Monogène, Ancyrocephalidae) parasite de Liza ramada (Risso, 1826) (Téléostéen, Mugilidae). Z. Parasitenkd. 52: 229-240.

LAMBERT A. 1977b: L'oncomiracidium d'Ancyrocephalus paradoxus Creplin, 1839 (Monogenea, Monopistho-

Received 21 June 2000 cotylea) parasite de Sander lucioperca (Téléostéen, Percidae). Ann. Parasitol. Hum. Comp. 52: 493-505.

LAMBERT A. 1978a: Précisions sur l'oncomiracidium de Tetraonchus monenteron (Wagener, 1857) Diesing, 1858 (Monogenea, Tetraonchidae) parasite d'Esox lucius L. (Téléostéen). Ann. Parasitol. Hum. Comp. 53: 117-121.

LAMBERT A. 1978b: Recherches sur l'oncomiracidium de Capsalidae (Monogenea). Ann. Parasitol. Hum. Comp. 53: 351-357.

LAMBERT A. 1979: Recherches sur les affinités phylétiques des vers parasites Gyrodactylidea (Monogenea). C. R. Acad. Sci., Paris 288, Serie D: 231-233.

LYNCH J.E. 1933: The miracidium of Heronimus chelydrae MacCallum. Q. J. Microsc. Sci. 76: 13-33.

MAEDER A.M. 1973: Monogènes et trématodes parasites d'amphibiens en Côte d'Ivoire. Rev. Suisse Zool. 80: 267322.

PETERS A. 1955a: Experiments on the mechanism of silver staining. Part I. Impregnation. Q. J. Microsc. Sci. 96: 84102.

PETERS A. 1955b: Experiments on the mechanism of silver staining. II. Development. Q. J. Microsc. Sci. 96: 103-115.

PRUDHOE S. 1957: Trematoda. In: Exploration du Parc National de l'Upemba. I. Mission G.F. de Witte en collaboration avec W. Adam, A. Janssens, L. Van Meel et R. Verheyen (1946-1949) 48: 1-28.

SHINN A.P., GIBSON D.I., SOMMERVILLE C. 1997: Argentophilic structures as a diagnostic criterion for the discrimination of species of the genus Gyrodactylus von Nordmann (Monogenea). Syst. Parasitol. 37: 47-57.

SHINN A.P., GIBSON D.I., SOMMERVILLE C. 1998a: Chaetotaxy of members of the Gyrodactylidae (Monogenea), with comments upon their systematic relationships with the Monopisthocotylea and Polyopisthocotylea. Syst. Parasitol. 39: 81-94.

SHINN A.P., GIBSON D.I., SOMMERVILLE C. 1998b: The application of chaetotaxy in the discrimination of Gyrodactylus salaris Malmberg, 1957 (Gyrodactylidae: Monogenea) from species of the genus parasitising British salmonids. Int. J. Parasitol. 28: 805-814.

SWITZER R.C., III 2000: Morphologic approaches for evaluating nervous system injury. Toxicol. Pathol. 28: 7083.

TINSLEY R.C. 1978: Oviposition, hatching and the oncomiracidium of Eupolystoma anterorchis (Monogenoidea). Parasitology 77: 121-132.

Accepted 5 January 2001 